\title{
Design and construction of the moving optical systems of IRAIT
}

\author{
Josep Colomé ${ }^{1}$, Carlos Abia ${ }^{2}$, Inma Domínguez ${ }^{2}$, Jordi Isern ${ }^{1,3}$, \\ Gino Tosti ${ }^{4}$, Maurizio Busso ${ }^{4}$, Giuliano Nucciarelli ${ }^{4}$, Fabio Roncella ${ }^{4}$, \\ Oscar Straniero ${ }^{5}$ and Mauro Dolci ${ }^{5}$, for the IRAIT Collaboration \\ ${ }^{1}$ Institut d'Estudis Espacials de Catalunya (IEEC), E-08034 Barcelona, Spain \\ email: colome@ieec.cat \\ ${ }^{2}$ Dpto.Física Teórica y del Cosmos, Universidad de Granada, E-18071 Granada, Spain \\ ${ }^{3}$ Institut de Ciències de l'Espai (CSIC), E-08034 Barcelona, Spain \\ ${ }^{4}$ Dipartimento di Fisica, Universitá di Perugia, via A. Pascoli, I-06100 Perugia, Italy \\ ${ }^{5}$ INAF-Osservatorio di Teramo, Via Maggini, I-64100 Teramo, Italy
}

\section{Introduction}

The IRAIT (International Robotic Antarctic Infrared Telescope) project (Tosti et al. 2006 ) is based on a $80 \mathrm{~cm}$ aperture telescope to observe in the infrared range. It is due to start operations in spring 2008, several months after installation in Dome C (Antarctica). We describe the contributions made to such project by the Institute for Space Studies of Catalonia (IEEC) and the University of Granada, whose participation has been mainly focused in developing the moving optical system for the secondary (M2) and tertiary (M3) mirrors of the telescope. Moving parts of the optical system provide focusing and chopping capabilities, implemented in M2, and a rotation mechanism, implemented in M3, allow observation in either Nasmyth foci. The work package includes the design and construction of both mirrors, the mechanical supports, the electronics and the control software, all prepared to work at the low temperatures at Antarctica. A Spanish company, NTE, was contracted to carry out the design and manufacture. Tests at low temperature and integration in the telescope were finished during summer 2006, before sending the telescope to Antarctica, scheduled by the end of the same year.

\section{Secondary mirror driver subsystem}

The M2 Drive Subsystem is the system in charge of providing the following movements to the mirror: (1) Focus: movement of the mirror in Uz axis, (2) Chopping: tip-tilt mirror movement. Three main parts compose the assembly: $(a)$ fixed subassembly; $(b)$ mobile subassembly or focuser; and $(c)$ chopper and M2 mirror subassembly. A number of parts are made of stainless steel in order to prevent corrosion or important dimensional variations caused by the coefficient of thermal expansion that could block the system, leaving it unusable at certain temperatures. Other materials are used: anodized aluminium, carbon fiber composite and Teflon.

(a) Fixed subassembly: holds the cryogenic motors and also contains the guiding system, the safety switches and the backward and forward actuator.

(b) Focuser: the focusing mechanism is realized by means of a linear actuator, manufactured by INA, which include a motor and a reduction stage (Pythron VSS32.200.1.2.UHVC and VPGL $32 \mathrm{i}-50$ UHVC units). The stroke of the actuator is $100 \mathrm{~mm}$, and the screw pitch is $2 \mathrm{~mm}$ per revolution. Two limit switches prevent the actuator from getting over 
the limits. These guiding systems have been considered as the most appropriate due to its compactness, high load capacity and accuracy on corrosion resistance. The cryogenic motor supplies the mechanical power to move this assembly over a temperature range that goes from $-270^{\circ} \mathrm{C}$ up to $30^{\circ} \mathrm{C}$. The rotational movement of the motor is converted into a lineal movement through a roller screw. The roller screw is manufactured on a G1 ISO quality corrosion proof that assures the accuracy, repeatability and steadiness of the advancing movement. The kinematics mechanism has been dimensioned for not being back drivable. All mobile parts are lubricated with cryogenic grease with melting point of $-90^{\circ} \mathrm{C}$ in order to avoid crystallization that would lead to a halt of the system.

(c) Chopper and mirror assembly: the chopper assembly is the mechanism that provides the tilting movement on the XY plane to the M2 mirror. The mechanism is designed to provide an angular oscillation from 0 to $\pm 4.6 \mathrm{mrad}$ (equivalent to $5^{\prime} \times 5^{\prime}$ in the sky) over the specified temperature range. This device allows us to obtain a maximum chopping frequency of $25 \mathrm{~Hz}$, compatible with the lower resonance frequency of the telescope top ring which was estimated to be about $80 \mathrm{~Hz}$. Within the mobile parts there are the piezoactuators, manufactured by PiezoMechanik technology, that provide the displacement to activate the chopping movement. They have cryogenic capabilities and are close loop servo actuated. The sensors that supply the feedback are Eddy current. The reason of this choice instead of a capacitance sensor is that they are more suitable for adverse environment conditions, such as moisture presence or possible ice deposition on the sensor surface. The chopper subassembly is composed for the following parts: radial spring, piezo stacks, mirror support, M2 mirror, sensor position, tilting adjusting system.

\section{Tertiary mirror driver subsystem}

The M3 Drive Subsystem disposes of the following performances: $(i)$ Locates the mirror in proper place to receive the light from M2 and send it to the cameras located at the Nasmyth focus. To obtain a correct position, two adjusting mechanisms have been implemented: Tilt Correction Mechanism, in base, and Mirror Position Mechanism, in mirror base; (ii) Allows mirror rotation in $z-z$ axis from 0 to $180^{\circ}$ with high accuracy; (iii) Allows fixation of the whole subsystem in the M3 Interface Area.

This subassembly can be divided in the next main parts and mechanisms:

(a) M3 Mirror: main element of the subsystem. It has an oval shape with a rear square interface to place and fix the element.

(b) Mirror position mechanism: mechanism in charge of adjusting the mirror orientation once the M3 Drive subsystem is assembled. It consists on a rear flexure, used to join the mirror and the tube, and one adjustment screw, used to modify the angle orientation.

(c) Tube: element in charge of providing stiffness to the subsystem. It is made of stainless steel and disposes of a lower flange to fix it to the rotating actuator and an upper flange to accommodate the mirror position mechanism. This upper flange is $45^{\circ}$ inclined to direct the M2 light to the Nasmyth focus.

(d) Rotating actuator: it is used to allow z-z rotation and $z-z$ adjustment of the subsystem is based on a Micos PRS-110 precision rotation stage modified for operation in a low-temperature environment (down to $-80^{\circ} \mathrm{C}$ ) The unit incorporates two electrical limit switches that will be set at the $0^{\circ}$ and $180^{\circ}$ positions. The repeatability of these switches $(5 \mu \mathrm{m})$ provides an angular repeatability of $0^{\circ} .008$.

(e) Axial alignment mechanism: mechanism located below the rotating actuator and it is able to rectify misalignment in the mirror due to mechanical tolerances. This mechanism can tilt the tube in two axis thanks to a platform driven by three screws. 
(f) Base: element in charge of the following issues: support all the subsystem with enough stiffness, fix all the assembly in the interface area, allow to plug and unplug the Rotating Actuator wires and connectors, allow to actuate the tilt correction mechanism, allow inner access for cleaning purposes. An Hex tool accommodation is placed in the base centre. It permits to drive the adjustment screw of the Mirror Position Mechanism in order to tilt the mirror.

\section{Acknowledgements}

This project is supported by the Spanish Ministry of Education and Science. IRAIT is a project approved by the ARENA consortium, network funded by the European Community's 6th Framework Programme. We acknowledge NTE S.A. and Marcon Telescopes for the outwork.

\section{Reference}

Tosti, G., Busso, M., Nucciarelli, G., et al., 2006, SPIE, 6267, 47 